\title{
Low energy QCD measurements and ChPT tests with Kaons at CERN
}

\author{
Monica Pepe ${ }^{1 *}$ \\ INFN Sezione di Perugia \\ Via A. Pascoli, 06123 Perugia, Italy \\ E-mail: monica.pepe@pg.infn.it
}

The NA48/2 experiment at CERN SPS collected data in 2003-2004 allowing several precise measurements in the charged kaon decay sector. This work presents preleminary measurements of the form factors of charged kaon semileptonic decays, based on 4.0 million $\mathrm{K}_{e 3}^{ \pm}\left(\mathrm{K}^{ \pm} \rightarrow \pi^{0} \mathrm{e}^{ \pm} v\right)$ and 2.5 million $\mathrm{K}_{\mu 3}^{ \pm}\left(\mathrm{K}^{ \pm} \rightarrow \pi^{0} \mu^{ \pm} v\right)$ decays, both with negligible background: the $\mathrm{K}_{e 3}^{ \pm}$and $\mathrm{K}_{\mu 3}^{ \pm}$ results are in good agreement allowing for a combined result which matches the precision of the current world average. Furthermore, the collaboration has collected large samples of $\mathrm{K}_{\mathrm{e} 4}{ }^{+-}$ $\left(\mathrm{K}^{ \pm} \rightarrow \pi^{+} \pi^{-} \mathrm{e}^{ \pm} v\right)$ and $\mathrm{K}_{\mathrm{e} 4}{ }^{00}\left(\mathrm{~K}^{ \pm} \rightarrow \pi^{0} \pi^{0} \mathrm{e}^{ \pm} v\right)$ decays. Using 1.1 million $\mathrm{K}_{\mathrm{e} 4}{ }^{+-}$and $66000 \mathrm{~K}_{\mathrm{e} 4}{ }^{00}$ decays an improved determination of the branching fraction and detailed form factor studies are performed. In the charged mode the measurement of the phase difference between the S- and Pwave states of the $\pi \pi$ system allows a precise determination of $a_{0}^{I}$, the isospin $\mathrm{I}=0$ and $\mathrm{I}=2 \mathrm{~S}$ wave $\pi \pi$ scattering lengths. A combination of this result with the NA48/2 measurement obtained in $\mathrm{K}_{3} \pi\left(\mathrm{K}^{ \pm} \rightarrow \pi^{0} \pi^{0} \pi^{ \pm}\right)$decays leads to a further improved determination of $a_{0}^{0}$ and the first precise experimental measurement of $a_{0}^{2}$.

XV International Conference on Hadron Spectroscopy (Hadron 2013)

November 4-8 2013

Nara, Japan

\footnotetext{
${ }^{1}$ Speaker

* On behalf of the NA48/2 Collaboration: G. Anzivino, R. Arcidiacono, W. Baldini, S. Balev, J.R. Batley, M. Behler, S. Bifani, C. Biino, A. Bizzeti, B. Bloch-Devaux, G. Bocquet, N. Cabibbo, M. Calvetti, N. Cartiglia, A. Ceccucci, P. Cenci, C. Cerri, C. Cheshkov, J.B. Chèze, M. Clemencic, G. Collazuol, F. Costantini, A. Cotta Ramusino,

D. Coward, D. Cundy, A. Dabrowski, P. Dalpiaz, C. Damiani, M. De Beer, J. Derré, H. Dibon, L. DiLella, N. Doble, K. Eppard, V. Falaleev, R. Fantechi, M. Fidecaro, L. Fiorini, M. Fiorini, T. Fonseca Martin, P.L. Frabetti,

L. Gatignon, E. Gersabeck, A. Gianoli, S. Giudici, A. Gonidec, E. Goudzovski, S. Goy Lopez, M. Holder, P. Hristov, E. Iacopini, E. Imbergamo, M. Jeitler, G. Kalmus, V. Kekelidze, K. Kleinknecht, V. Kozhuharov, W. Kubischta, G. Lamanna, C. Lazzeroni, M. Lenti, L. Litov, D. Madigozhin, A. Maier, I. Mannelli, F. Marchetto, G. Marel, M. Markytan, P. Marouelli, M. Martini, L. Masetti, E. Mazzucato, A. Michetti, I. Mikulec, N. Molokanova, E. Monnier, U. Moosbrugger, C. Morales Morales, D.J. Munday, A. Nappi, G. Neuhofer, A. Norton, M. Patel, M. Pepe, A. Peters, F. Petrucci, M.C. Petrucci, B. Peyaud, M. Piccini, G. Pierazzini, I. Polenkevich,

Yu. Potrebenikov, M. Raggi, B. Renk, P. Rubin, G. Ruggiero, M. Savrié, M. Scarpa, M. Shieh, M.W. Slater, M. Sozzi, S. Stoynev, E. Swallow, M. Szleper, M. Valdata-Nappi, B. Vallage, M. Velasco, M. Veltri, S. Venditti, M. Wache, H. Wahl, A. Walker, R. Wanke, L. Widhalm, A. Winhart, R. Winston, M.D. Wood, S.A. Wotton, A. Zinchenko, M. Ziolkowski.
} 


\section{Introduction}

The main goal of the NA48/2 experiment, collecting data in 2003-2004 at CERN SPS, was the search for direct $\mathrm{CP}$ violation in the decay of $\mathrm{K}^{ \pm}$into three pions [1]. The large statistics accumulated has allowed the study of a variety of rare kaon decay modes; here recent experimental results on charged kaon semileptonic decays from NA48/2 are presented.

\section{The NA48/2 experiment}

Simultaneous $\mathrm{K}^{+}$and $\mathrm{K}^{-}$beams are produced in the same beryllium target by impinging $400 \mathrm{GeV}$ protons from the CERN/SPS accelerator. The beam momentum range of $(60 \pm 3)$ $\mathrm{GeV} / \mathrm{c}$ is selected by two front-end "achromats" and focused $\sim 200 \mathrm{~m}$ downstream, at the end of the decay region. The main components of the NA48 detector, described in detail in Ref.[2], are: a magnetic spectrometer consisting of two sets of drift chambers and a central dipole magnet, providing a momentum resolution $\sigma(p) / p=(1.02 \oplus 0.044 \cdot p) \%(p$ in $\mathrm{GeV} / c)$; a hodoscope consisting of two planes of plastic scintillators segmented into horizontal and vertical strips providing a fast trigger for charged particles with very good $(\sim 150 \mathrm{ps})$ time resolution; a liquid Krypton calorimeter (LKr), 27 radiation lengths deep, used to measure electromagnetic energy deposition of photons and electrons with a resolution $\sigma(E) / E=(3.2 / \sqrt{ } E$ $\oplus 9.0 / E \oplus 0.42) \%(E$ in $\mathrm{GeV})$; a muon detector system, consisting of three segmented scintillator planes each shielded by a $80 \mathrm{~cm}$ thick iron wall, to identify muons in $\mathrm{K}_{\mu 3}^{ \pm}$events. The energy/momentum (E/p) ratio is used for electron/pion $(\mathrm{e} / \pi)$ discrimination.

\section{Semileptonic $K^{ \pm}{ }_{\ell 3}$ decays}

Semileptonic kaon decays offer the theoretically cleanest access to the CKM matrix element $\left|\mathrm{V}_{\text {us }}\right|$ and the most accurate test of the CKM matrix unitarity. The physical quantity used to extract $\left|\mathrm{V}_{\text {us }}\right|$ experimentally is the photon inclusive $\mathrm{K}_{\ell 3}^{ \pm}(\ell=e$ or $\mu)$ decay rate and besides inputs from theory, now calculated with a precision of $0.5 \%$, and small radiative and SU(2) corrections, one needs to compute phase space integrals including form factor measurements.

The hadronic matrix element of the $\mathrm{K}_{\ell 3}^{ \pm}$decays is described by two dimensionless form factors $(\mathrm{FF}) \mathrm{f}_{ \pm}(\mathrm{t})$ which depend on the squared four-momentum $t$ transferred to the $\ell-v$ system. They can be expressed in terms of vector $f_{+}(t)$ and scalar $f_{0}(t)$ exchange contributions, parametrized either as a Taylor expansion ("quadratic parametrization") with coefficients $\lambda_{+, 0}^{\prime}$ and $\lambda^{\prime \prime}{ }_{+, 0}$ (slope and curvature), or by assuming the dominance of vector (V) or scalar (S) resonance exchange ("pole parametrization"), where pole masses are the only free parameters. Only the vector $\mathrm{FF}$ is measurable in $\mathrm{K}_{\mathrm{e} 3}^{ \pm}$(due to lepton mass suppression in the scalar $\mathrm{FF}$ amplitude) while both vector and scalar $\mathrm{FF}$ are accessible in $\mathrm{K}_{\mu 3}^{ \pm}$. As the vector $\mathrm{FF} \mathrm{f}_{+}(0)$ at 0 momentum transfer cannot be measured directly, FF normalized to $f_{+}(0)$ are considered.

The NA48/2 results on FF are obtained using data collected during a dedicated three-day run with minimum bias trigger in 2004. Four million $\mathrm{K}^{ \pm}{ }_{\mathrm{e} 3}$ and 2.5 million $\mathrm{K}_{\mu 3}^{ \pm}$events were fully 
reconstructed with sub-percent background contamination. After background subtraction, acceptance and radiative corrections, the Dalitz plot of the decay is fitted with a procedure which masks the bins at the border of the kinematical allowed region, extracting the FF for both final states and both parametrizations. Since results for both channels agree well with each other (for both parametrizations) they may be combined to give the preliminary results shown in Table 1.

\begin{tabular}{|c|c|c|c|}
\hline Quadratic $\left(\times 10^{3}\right)$ & $\lambda^{\prime}{ }_{+}$ & $\lambda^{\prime \prime}+$ & $\lambda_{0}$ \\
\hline $\mathrm{K}_{\mathrm{e} 3}^{ \pm} \mathrm{K}_{\mu 3}^{ \pm}$combined & $26.98 \pm 1.11$ & $0.81 \pm 0.46$ & $16.23 \pm 0.95$ \\
\hline Pole $\left(\mathrm{MeV} / \mathrm{c}^{2}\right)$ & $m_{V}$ & & $m_{S}$ \\
\hline $\mathrm{K}_{\mathrm{e} 3}^{ \pm} \mathrm{K}_{\mu 3}^{ \pm}$combined & $877 \pm 6$ & & $1176 \pm 31$ \\
\hline
\end{tabular}

Table 1. Preliminary NA48/2 results for $\mathrm{K}_{\mathrm{e} 3}^{ \pm}$and $\mathrm{K}_{\mu 3}^{ \pm}$combined form factors.

Figure 1 compares the combined quadratic fit results reported by recent experiments: $68 \%$ confidence level contours are shown for both neutral (KLOE, KTeV and NA48) and charged (NA48/2, ISTRA+ only studied $\mathrm{K}^{-}$) kaons. The NA48/2 results agree with other experiments and match the precision of the 2010 FlaviaNet [3] world average. The combined $\mathrm{K}_{\mathrm{e} 3}^{ \pm}$and $\mathrm{K}_{\mu 3}^{ \pm}$ results from NA48/2 are the first high precision FF measurement with both $\mathrm{K}^{+}$and $\mathrm{K}^{-}$mesons.

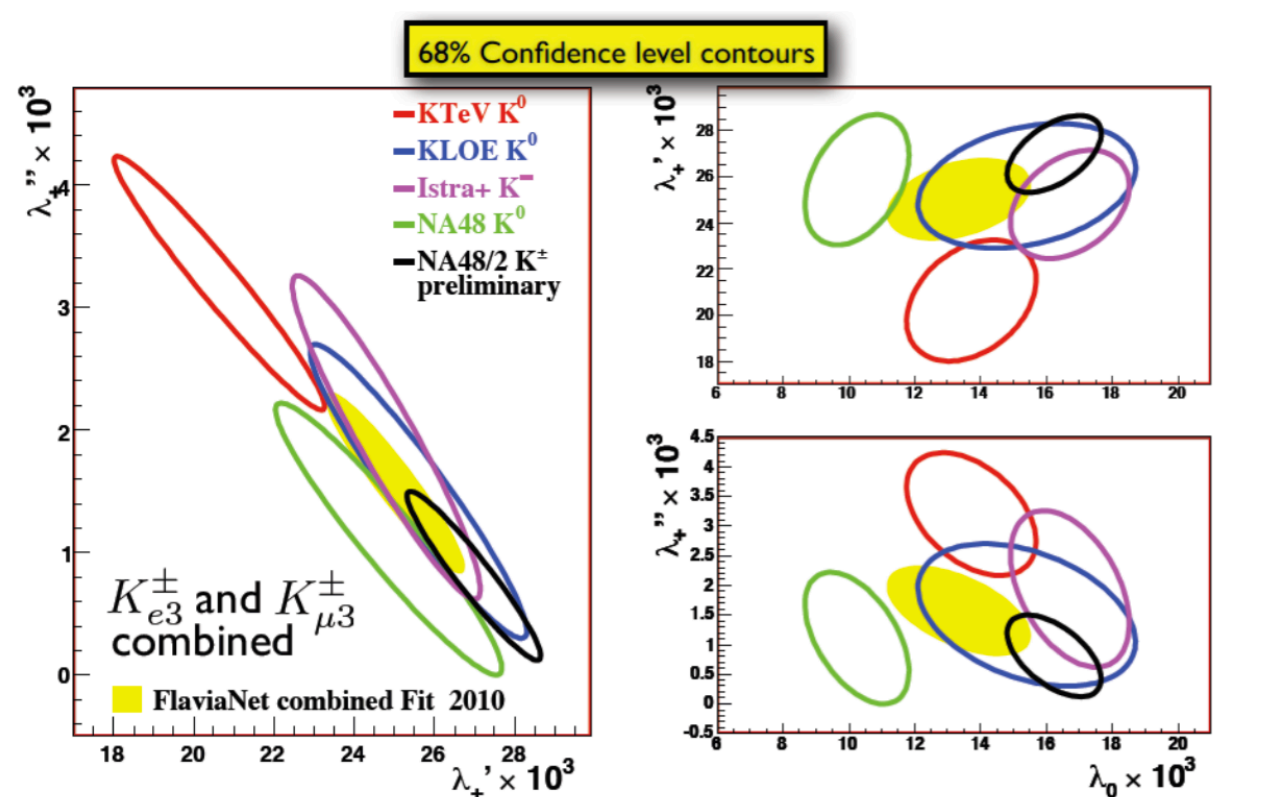

Figure 1. Preliminary combined quadratic fit results for $\mathrm{K}_{\ell 3}$ decays. Ellipses are $68 \% \mathrm{CL}$ contours.

\section{Semileptonic $\mathbf{K}_{\text {e4 }}^{ \pm}$decays}

The study of $\mathrm{K}_{\mathrm{e} 4}$ decays is of particular interest providing access to two-pion final state interaction in a clean environment given the absence of any other hadron.

Four-body final state decays are fully described by five kinematic variables, the CabibboMaksymowicz variables: two invariant masses $S_{\pi}=M^{2} \pi$ and $S_{\mathrm{e}}=M^{2}$ ev and three angles $\theta_{\pi}, \theta_{e}$ and $\varphi$. The hadronic complex form factors (FF) can be developed in a partial wave expansion: when limiting the expansion to S- and $\mathrm{P}$-waves and considering a unique phase $\delta_{p}$ for all $\mathrm{P}$-wave $\mathrm{FF}$, two axial FF $(F, G)$ and one vector $\mathrm{FF}(H)$ contribute to the transition amplitude. The 
differential rate in the 5-dimensional space depends on five real numbers, four $\mathrm{FF}\left(F_{s}, F_{p}, G_{p}\right.$, $\left.H_{p}\right)$ and one phase difference $\left(\delta=\delta_{s}-\delta_{p}\right)$ that are measured together with their energy variation with $S_{\pi}$ and $S e$. For the neutral mode $\mathrm{K}_{\mathrm{e} 4}{ }^{00}$ the formalism is simpler due to Bose statistics and the FF reduce to $F_{s}$ only.

\subsection{Form Factors and Branching Ratio}

After a similar event reconstruction and selection, $1.1 \times 10^{6} \mathrm{~K}_{\mathrm{e} 4}{ }^{+-}$and $6.6 \times 10^{4} \mathrm{~K}_{\mathrm{e} 4}{ }^{00}$ signal events are found with background contamination at percent level $[4,5]$. The FF and branching ratios (BR), measured with unprecedented precision, are published in [4] for $\mathrm{K}_{\mathrm{e} 4}{ }^{+-}$ while the $\mathrm{K}_{\mathrm{e} 4}{ }^{00}$ results are still preliminary. The BR (inclusive of $\mathrm{K}_{\mathrm{e} 4 \gamma}$ ) measured relative to the abundant $\mathrm{K}_{3} \pi$ modes $\left(\mathrm{K}^{ \pm} \rightarrow \pi^{+} \pi^{-} \pi^{ \pm}\right.$and $\left.\mathrm{K}^{ \pm} \rightarrow \pi^{0} \pi^{0} \pi^{ \pm}\right)$are listed in Table 2: the external uncertainties from the normalization mode BR measurements are presently the dominant errors.

\begin{tabular}{|c|c|c|}
\hline Decay Mode & BR & PDG value \\
\hline $\mathbf{K}_{\mathrm{e} 4}{ }^{+-}$ & $\left(4.257 \pm 0.004_{\text {stat }} \pm 0.016_{\text {syst }} \pm 0.031_{\text {ext }}\right) \times 10^{-5}$ & $(4.09 \pm 0.10) \times 10^{-5}$ \\
\hline $\mathbf{K}_{\mathrm{e} 4}{ }^{\mathbf{0 0}}$ & $\left(2.585 \pm 0.010_{\text {stat }} \pm 0.010_{\text {syst }} \pm 0.032_{\text {ext }}\right) \times 10^{-5}$ & $(2.2 \pm 0.4) \times 10^{-5}$ \\
\hline
\end{tabular}

Table 2. NA48/2 measured BR (inclusive of $\mathrm{K}_{\mathrm{e} 4} \gamma$ ) for $\mathrm{K}_{\mathrm{e} 4}{ }^{+-}$and $\mathrm{K}_{\mathrm{e} 4}{ }^{00}$ decays.

The energy dependence of the FF was studied in the plane $\left(S_{\pi}, S_{\mathrm{e}}\right)$ adjusting the differential distributions of simulated events to those of data in small equally populated bins of the multidimensional kinematic space. The $F_{s}$ form factor variation with $q^{2}=\left(S_{\pi} / 4 m^{2}-1\right)$ is shown in Fig. 2 where the same quadratic behaviour is visible in both modes at $q^{2}>0$. The deficit of events below the $2 m_{\pi^{+}}$threshold in the $\mathrm{K}_{\mathrm{e} 4}{ }^{00}$ data can be explained by charge exchange rescattering effects in the $\pi \pi$ system as observed in $\mathrm{K}_{3 \pi}$ decays [6].
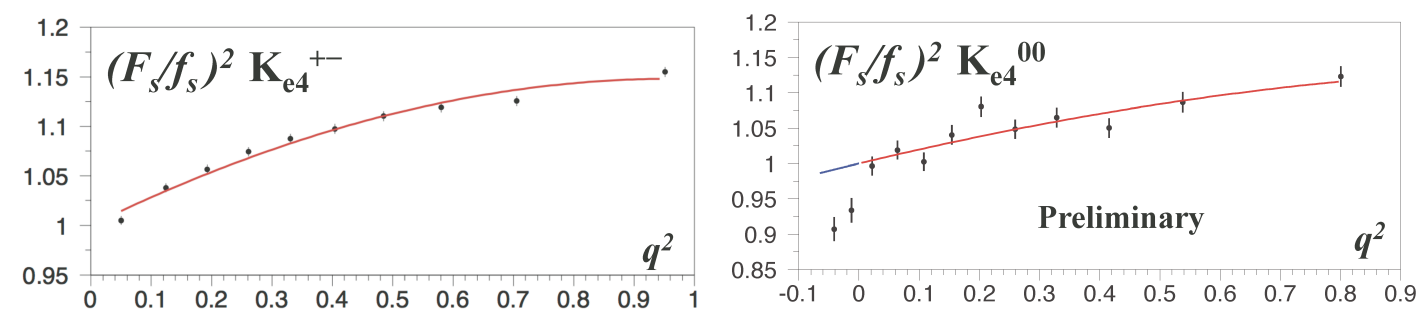

Figure 2. Relative $\mathrm{FF}\left(\mathrm{F}_{\mathrm{s}} / \mathrm{f}_{\mathrm{s}}\right)^{2}$ variation with $\mathrm{q}^{2}$ for $\mathrm{K}_{\mathrm{e} 4}{ }^{+-}$(left) and $\mathrm{K}_{\mathrm{e} 4}{ }^{00}$ (right) decays. The red lines for $\mathrm{q}^{2}>0$ are degree-2 polynomial fits to data, while the blue line at $\mathrm{q}^{2}<0$ in the right plot is the extrapolation to negative values.

\subsection{Scattering lengths from $K_{\mathrm{e} 4}$ and $K_{3 \pi}$ decays}

The $\pi \pi$ scattering lengths are among the most precise predictions of Chiral Perturbation Theory (ChPT). NA48/2 has performed precise measurements using $\mathrm{K}_{\mathrm{e} 4}{ }^{+-}$and $\mathrm{K}_{3 \pi}$ decays. $\mathrm{K}_{3} \pi$ decays are a sensitive probe for S-wave scattering lengths $a_{0}^{I}$ through final state scattering effects resulting in a cusp like structure of the $\pi \pi$ invariant mass distribution at the $2 m_{\pi^{+}}$ threshold. NA48/2 has reconstructed 60 million such events and determined $a_{0}^{I}$ from the shape of the $M^{2} \pi$ distribution [6]. $\mathrm{K}_{\mathrm{e} 4}{ }^{+-}$decays have small BR (1.1 million events reconstructed by NA48/2) but allow the study of final state interaction of two pions in a clean hadronic environment. Scattering lengths are extracted from the measured phase shift after applying 
isospin corrections [4]. These two different but complementary approaches to $\pi \pi$ scattering near threshold are used to extract S-wave scattering lengths $a_{0}^{I}$ corresponding to isospin $\mathrm{I}=0$ and $\mathrm{I}=2$.

As $\mathrm{K}_{3 \pi}$ and $\mathrm{K}_{\mathrm{e} 4}$ results come from statistically independent analyses, they can be combined (neglecting the potential but small common systematic uncertainties) in a more precise result:

$$
\begin{aligned}
a_{0}^{0}=0.2210 \pm 0.0047_{\text {stat }} \pm 0.0040_{\text {syst }} \\
a_{0}^{2}=-0.0429 \pm 0.0044_{\text {stat }} \pm 0.0028_{\text {syst }} \\
a_{0}^{0}-a_{0}^{2}=0.2639 \pm 0.0020_{\text {stat }} \pm 0.0015_{\text {syst }}
\end{aligned}
$$

Figure 3 compares the NA48/2 measurements to recent experimental results from DIRAC [7], precise calculations from lattice QCD [8] and most elaborate predictions from ChPT [9].
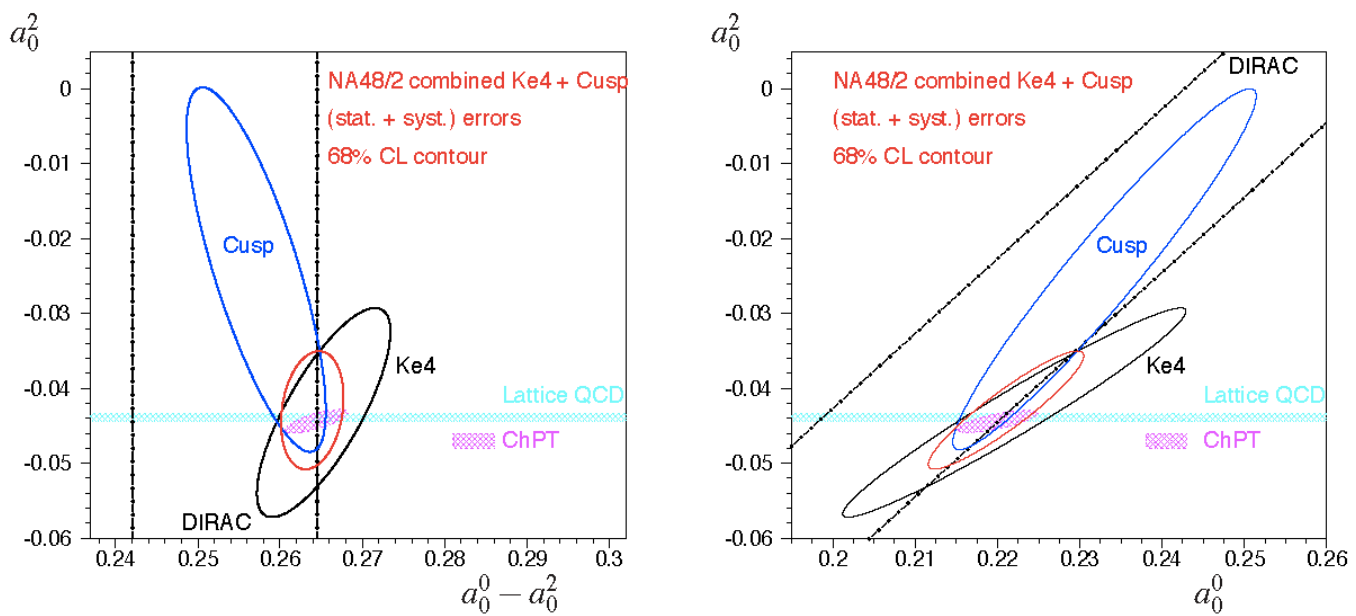

Figure 3. $N A 48 / 2 K_{3} \pi$ (blue) and $K_{e 4}$ (black) results in the $\left(a_{0}^{0}-a_{0}^{2}, a_{0}^{2}\right)$ (left) and $\left(a_{0}^{0}, a_{0}^{2}\right)$ (right) planes. Red contour is the combined NA48/2 result compared to DIRAC result band and lattice QCD and ChPT predictions.

\section{References}

[1] NA48/2: J.R. Batley et al., Eur.Phys.J. C52 (2007) 875.

[2] NA48/2: V. Fanti et al., Nucl.Instrum.Meth. A574 (2007) 433.

[3] M. Antonelli et al., Eur. Phys. J. C69 (2010) 399.

[4] NA48/2: J.R. Batley et al., Eur. Phys. J. C70 (2010) 635; ibidem, Phys. Lett. B715 (2012) 105.

[5] B. Bloch-Devaux, PoS (KAON13) 028.

[6] NA48/2: J.R. Batley et al., Eur. Phys. J. C64 (2009) 589.

[7] DIRAC: B. Adeva et al., Phys. Lett. B704 (2011) 24.

[8] S. Beane et al., Phys. Rev. D77 (2008) 014505 ; X. Feng et al., Phys. Lett. B684 (2010) 268.

[9] G. Colangelo et al., Phys. Lett. B488 (2000) 261; ibidem, Phys. Rev. Lett. 86 (2001) 5008. 